\title{
Applying Bayesian Neural Networks to Separate Neutrino Events from Backgrounds in Reactor Neutrino Experiments
}

\author{
Ye Xü, Yixiong Meng ${ }^{a}$, Weiwei $X u^{a}$
}

${ }^{a}$ Department of Physics, Nankai University, Tianjin 300071, People's Republic of China

\begin{abstract}
A toy detector has been designed to simulate central detectors in reactor neutrino experiments in the paper. The samples of neutrino events and three major backgrounds from the Monte-Carlo simulation of the toy detector are generated in the signal region. The Bayesian Neural Networks(BNN) are applied to separate neutrino events from backgrounds in reactor neutrino experiments. As a result, the most neutrino events and uncorrelated background events in the signal region can be identified with BNN, and the part events each of the fast neutron and ${ }^{8} \mathrm{He} /{ }^{9} \mathrm{Li}$ backgrounds in the signal region can be identified with BNN. Then, the signal to noise ratio in the signal region is enhanced with BNN. The neutrino discrimination increases with the increase of the neutrino rate in the training sample. However, the background discriminations decrease with the decrease of the background rate in the training sample.
\end{abstract}

Keywords: Bayesian neural networks, neutrino oscillation, identification

PACS numbers: 07.05.Mh, 29.85.Fj, 14.60.Pq

\section{Introduction}

The main goals of reactor neutrino experiments are to detect $\overline{\nu_{e}} \rightarrow \overline{\nu_{x}}$ oscillation and precisely measure the mixing angle of neutrino oscillation $\theta_{13}$. The experiment is designed to detect reactor $\overline{\nu_{e}}$ 's via the inverse $\beta$-decay reaction

$$
\overline{\nu_{e}}+p \rightarrow e^{+}+n
$$

The signature is a delayed coincidence between $e^{+}$and the neutron captured signals. In the paper, only three important sources of backgrounds are taken into account and they are the uncorrelated background from natural radioactivity and the correlated backgrounds from fast neutrons and ${ }^{8} \mathrm{He} /{ }^{9} \mathrm{Li}$. The backgrounds like the neutrino events consist of two signals, a fast signal and a delay signal. It

\footnotetext{
${ }^{*}$ Corresponding author, e-mail address: xuye76@nankai.edu.cn
} 
is vital to separate neutrino events from backgrounds accurately in the reactor neutrino experiments. The selection of the neutrino events based on the cuts is a methods that the event space is divided into two regions by a hyper-cuboid based on the cuts, and the events inside the hyper-cuboid, called the signal region, are regarded as neutrino events and the events outside the hyper-cuboid are regarded as backgrounds. In fact, the backgrounds in the signal region couldn't be rejected by the method. The Bayesian neural networks (BNN)[1] is an algorithm of the neural networks trained by Bayesian statistics. It is not only a non-linear function as neural networks, but also controls model complexity. So its flexibility makes it possible to discover more general relationships in data than the traditional statistical methods and its preferring simple models make it possible to solve the over-fitting problem better than the general neural networks[2]. BNN has been used to particle identification and event reconstruction in the experiments of the high energy physics, such as Ref.[3, 4, 5]. In this paper, BNN will be applied to discriminate the neutrino events from the background events in the signal region in the reactor neutrino experiments.

\section{The Classification with BNN[1, 5]}

The idea of Bayesian neural networks is to regard the process of training a neural network as a Bayesian inference. Bayes' theorem is used to assign a posterior density to each point, $\bar{\theta}$, in the parameter space of the neural networks. Each point $\bar{\theta}$ denotes a neural network. In the method of the Bayesian neural network, one performs a weighted average over all points in the parameter space of the neural network, that is, all neural networks. The methods make use of training data $\left(x_{1}, t_{1}\right),\left(x_{2}, t_{2}\right), \ldots,\left(x_{n}, t_{n}\right)$, where $\mathrm{t}_{i}$ is the known label associated with data $x_{i} . t_{i}=0,1, \ldots N-1$, if there are $N$ classes in the problems of classification; $x_{i}$ has $P$ components if there are $P$ factors on which the classification is influenced. That is the set of data $x=\left(x_{1}, x_{2}, \ldots, x_{n}\right)$ which corresponds to the set of target $t=\left(t_{1}, t_{2}, \ldots, t_{n}\right)$.The posterior density assigned to the point $\bar{\theta}$, that is, to a neural network, is given by Bayes' theorem

$$
p(\bar{\theta} \mid x, t)=\frac{p(x, t \mid \bar{\theta}) p(\bar{\theta})}{p(x, t)}=\frac{p(t \mid x, \bar{\theta}) p(x \mid \bar{\theta}) p(\bar{\theta})}{p(t \mid x) p(x)}=\frac{p(t \mid x, \bar{\theta}) p(\bar{\theta})}{p(t \mid x)}
$$

where data $x$ do not depend on $\bar{\theta}$, so $p(x \mid \theta)=p(x)$. We need the likelihood $p(t \mid x, \bar{\theta})$ and the prior density $p(\bar{\theta})$, in order to assign the posterior density $p(\bar{\theta} \mid x, t)$ to a neural network defined by the point $\bar{\theta} \cdot p(t \mid x)$ is called evidence and plays the role of a normalizing constant, so we ignore the evidence. That is,

$$
\text { Posterior } \propto \text { Likelihood } \times \text { Prior }
$$

We consider a class of neural networks defined by the function

$$
y(x, \bar{\theta})=\frac{1}{1+\exp [-s(x, \bar{\theta})]}
$$


where

$$
s(x, \bar{\theta})=b+\sum_{j=1}^{H} v_{j} \tanh \left(a_{j}+\sum_{i=1}^{P} u_{i j} x_{i}\right)
$$

The neural networks have $P$ inputs, a single hidden layer of $H$ hidden nodes and a single output. In the particular Bayesian neural networks described here, each neural network has the same structure. The parameter $u_{i j}$ and $v_{j}$ are called the weights and $a_{j}$ and $b$ are called the biases. Both sets of parameters are generally referred to collectively as the weights of the Bayesian neural networks, $\bar{\theta} . y(x, \bar{\theta})$ is the probability that an event, $(x, t)$, belongs to the signal. So the likelihood of $n$ training events is

$$
p(t \mid x, \bar{\theta})=\prod_{i=1}^{n} y^{t_{i}}(1-y)^{1-t_{i}}
$$

where it has been assumed that the events are independent with each other.

We get the likelihood, meanwhile we need the prior to compute the posterior density. But the choice of prior is not obvious. However, experience suggests a reasonable class is the priors of Gaussian class centered at zero, which prefers smaller rather than larger weights, because smaller weights yield smoother fits to data. In the paper, a Gaussian prior is specified for each weight using the Bayesian neural networks package of Radford Neal ${ }^{1}$. However, the variance for weights belonging to a given group(either input-to-hidden weights $\left(u_{i j}\right)$, hidden -biases $\left(a_{j}\right)$, hidden-to-output weights $\left(v_{j}\right)$ or output-biases $\left.(b)\right)$ is chosen to be the same: $\sigma_{u}^{2}, \sigma_{a}^{2}, \sigma_{v}^{2}, \sigma_{b}^{2}$, respectively. However, since we don't know, a priori, what these variances should be, their values are allowed to vary over a large range, while favoring small variances. This is done by assigning each variance a gamma prior

$$
p(z)=\left(\frac{\alpha}{\mu}\right)^{\alpha} \frac{z^{\alpha-1} e^{-z \frac{\alpha}{\mu}}}{\Gamma(\alpha)}
$$

where $z=\sigma^{-2}$, and with the mean $\mu$ and shape parameter $\alpha$ set to some fixed plausible values. The gamma prior is referred to as a hyperprior and the parameter of the hyperprior is called a hyperparameter.

Then, the posterior density, $p(\bar{\theta} \mid x, t)$, is gotten according to Eqs. (2),(5) and the prior of Gaussian distribution. Given an event with data $x^{\prime}$, an estimate of the probability that it belongs to the signal is given by the weighted average

\footnotetext{
${ }^{1}$ R. M. Neal, Software for Flexible Bayesian Modeling and Markov Chain Sampling, http://www.cs.utoronto.ca/ radford/fbm.software.html
} 


$$
\bar{y}\left(x^{\prime} \mid x, t\right)=\int y\left(x^{\prime}, \bar{\theta}\right) p(\bar{\theta} \mid x, t) d \bar{\theta}
$$

Currently, the only way to perform the high dimensional integral in Eq. (7) is to sample the density $p(\bar{\theta} \mid x, t)$ with the Markov Chain Marlo Carlo (MCMC) method $[1,6,7,8]$. In the MCMC method, one steps through the $\bar{\theta}$ parameter space in such a way that points are visited with a probability proportional to the posterior density, $p(\bar{\theta} \mid x, t)$. Points where $p(\bar{\theta} \mid x, t)$ is large will be visited more often than points where $p(\bar{\theta} \mid x, t)$ is small.

Eq. (7) approximates the integral using the average

$$
\bar{y}\left(x^{\prime} \mid x, t\right) \approx \frac{1}{L} \sum_{i=1}^{L} y\left(x^{\prime}, \bar{\theta}_{i}\right)
$$

where $L$ is the number of points $\bar{\theta}$ sampled from $p(\bar{\theta} \mid x, t)$. Each point $\bar{\theta}$ corresponds to a different neural network with the same structure. So the average is an average over neural networks, and the probability of the data $x^{\prime}$ belongs to the signal. The average is closer to the real value of $\bar{y}\left(x^{\prime} \mid x, t\right)$, when $L$ is sufficiently large.

\section{Toy Detector and Simulation[4]}

\subsection{Toy Detector}

In the paper, a toy detector is designed to simulate central detectors in the reactor neutrino experiments, such as Daya Bay experiment[9] and Double Chooz experiment[10], with CERN GEANT4 package[11]. The toy detector consists of three regions, and they are the Gd-doped liquid scintillator(Gd-LS from now on), the normal liquid scintillator(LS from now on) and the oil buffer, respectively. The toy detector of cylindrical shape like the detector modules of Daya Bay experiment and Double Chooz experiment is designed in the paper. The diameter of the Gd-LS region is 2.4 meter, and its height is 2.6 meter. The thickness of the LS region is 0.35 meter, and the thickness of the oil part is 0.40 meter. In the paper, the Gd-LS and LS are the same as the scintillator adopted by the proposal of the CHOOZ experiment[11]. The 8-inch photomultiplier tubes (PMT from now on) are mounted on the inside the oil region of the detector. A total of 366 PMTs are arranged in 8 rings of 30 PMTs on the lateral surface of the oil region, and in 5 rings of 24, 18, 12, 6, 3 PMTs on the top and bottom caps.

\subsection{Monte-Carlo Simulation of Toy Detector}

The response of the neutrino and background events deposited in the toy detector is simulated with GEANT4. Although the physical properties of the scintillator and the oil (their optical attenuation length, refractive index and so on) are wavelength dependent, only averages[11] (such as the optical attenuation length of Gd-LS with a uniform value is 8 meter and the one of LS is 20 meter) are used in 
the detector simulation. The program couldn't simulate the real detector response, but this won't affect the result of the comparison between BNN and the method based on the cuts.

According to the anti-neutrino interaction in the detector of the reactor neutrino experiments[12], the neutrino events are uniformly generated throughout Gd-LS region (see Fig. 1). The uncorrelated background events are generated in such a way that the fast signal energies are generated on the base of the energy distribute of the natural radioactivity in the proposal of the Day Bay experiment[9], the energies for the neutron events of the single signal are regarded as the delay signal energies, the delay times are uniformly generated from $2 \mu$ s to $100 \mu \mathrm{s}$ and the positions of the fast signal and the delay signal are uniformly generated throughout GD-LS region. The fast neutron events are uniformly generated throughout Gd-LS region and their energy are uniformly generated from $0 \mathrm{MeV}$ to $50 \mathrm{MeV}$, therein the events of two signals are regarded as the fast neutron backgrounds. Since the behavior of ${ }^{8} \mathrm{He} /{ }^{9} \mathrm{Li}$ decay in the detector couldn't be simulated by the Geant4 package, ${ }^{8} \mathrm{He} /{ }^{9} \mathrm{Li}$ events are generated in such a way that the fast signal energies are generated on the base of the energy distribute of ${ }^{8} \mathrm{He} /{ }^{9} \mathrm{Li}$ in the proposal of the Day Bay experiment[9], and the other physical quantities are from fast neutron events in the paper.

\section{Event Reconstruction[4]}

The task of the event reconstruction in the reactor neutrino experiments is to reconstruct the energy and the vertex of a signal. The maximum likelihood method (MLD) is a standard algorithm of the event reconstruction in the reactor neutrino experiments. The likelihood is defined as the joint Poisson probability of observing a measured distribution of photoelectrons over the all PMTs for given $(E, \vec{x})$ coordinates in the detector. The Ref.[13] for the work of the CHOOZ experiment shows the method of the reconstruction in detail.

In the paper, the event reconstruction with the MLD are performed in the similar way with the CHOOZ experiment[13], but the detector is different from the detector of the CHOOZ experiment, so compared to Ref.[13], there are some different points in the paper:

(1) The detector in the paper consists of three regions, so the path length from a signal vertex to the PMTs consist of three parts, and they are the path length in Gd-LS region, the one in LS region, and the one in oil region, respectively.

(2) Considered that not all PMTs in the detector can receive photoelectrons when a electron is deposited in the detector, the $\chi^{2}$ equation is modified in the paper and different from the one in the CHOOZ experiment, that is, $\chi^{2}=$ $\sum_{N_{j}=0} \bar{N}_{j}+\sum_{N_{j} \neq 0}\left(\bar{N}_{j}-N_{j}+N_{j} \log \left(\frac{N_{j}}{N_{j}}\right)\right)$, where $N_{j}$ is the number of photoelectrons received by the j-th PMT and $\bar{N}_{j}$ is the expected one for the j-th PMT[13].

(3) $c_{E} \times N_{\text {total }}$ and the coordinates of the charge center of gravity for the all visible photoelectrons from a signal are regarded as the starting values for the fit parameters $(E, \vec{x})$, where $N_{\text {total }}$ is the total numbers of the visible photoelectrons from a signal and $c_{E}$ is the proportionality constant of the energy $E$, that is, $E=c_{E} \times N_{\text {total }} . c_{E}$ is obtained through fitting $N_{\text {total }}$ 's of the $1 \mathrm{MeV}$ electron events, and is $\frac{1}{235 / \mathrm{MeV}}$ in the paper.

The fast and delay signals of a event in the toy detector are reconstructed using MLD, respectively. 


\section{Monte-Carlo Sample in Signal Region}

The selections of neutrino events are as follows:

(1) Positron energy: $1.3 \mathrm{MeV}<E_{e^{+}}<8 \mathrm{MeV}$;

(2) Neutron energy: $6 \mathrm{MeV}<E_{n}<10 \mathrm{MeV}$;

(3) Neutron delay: $2 \mu \mathrm{s}<\Delta t_{e^{+} n}<100 \mu \mathrm{s}$;

(4) Relative positron-neutron distance: $d_{e^{+} n}<100 \mathrm{~cm}$.

A hyper-cuboid in the event space is defined by the selection, and the inside is the signal region and the outside is the background region. 39000 events of neutrino are generated in the signal region. 11000 events each of uncorrelated background, fast neutrons and ${ }^{8} \mathrm{He} /{ }^{9} \mathrm{Li}$ are generated in the signal region, respectively.

\section{Neutrino Discrimination with BNN in Signal Region}

The energies of the fast signal and the delay $\operatorname{signal}\left(E_{e^{+}}, E_{n}\right)$, the delay time of the delay signal $\left(\Delta t_{e^{+} n}\right)$ and the distance between the fast signal and the delay signal $\left(d_{e^{+} n}\right)$ are used as inputs to all neural networks, which have the same structure. In the paper, all the networks have the input layer of four inputs, the single hidden layer of nine nodes and the output layer of a single output which is just the probability that an event belongs to the neutrino event. A Markov chain of neural networks is generated using the Bayesian neural networks package of Radford Neal, with a training sample consisting of the neutrino events and the backgrounds. One thousand iterations, of twenty MCMC steps each, are used. The neural network parameters are stored after each iteration, since the correlation between adjacent steps is very high. That is, the points in neural network parameter space are saved to lessen the correlation after twenty steps here. It is also necessary to discard the initial part of the Markov chain because the correlation between the initial point of the chain and the points of the part is very high. The initial three hundred iterations are discarded here. 3000 events each of the neutrino and the three backgrounds are used to test the identification capability of the trained BNN. In the paper, the BNNs are trained by the different training samples, which consist of the neutrino events and three backgrounds at different rates, since the different identification efficiencies are obtained with those BNNs. The results of the identification with those BNNs are listed in Tab. 1.

\section{Results and Discussion}

As Tab. 1, the neutrino discrimination increases from $82.6 \%$ to $91.2 \%$ with the increase of the neutrino rate from one second to nine fourteenth in the training sample using BNN in signal region. However, the background discriminations decrease with the decrease of the background rate in the training sample. The uncorrelated background discrimination decrease from $88.2 \%$ to $73.6 \%$ with the decrease of its rate from one sixth to one fourteenth in the training sample. The fast neutron background discrimination decreases from $48.5 \%$ to $37.6 \%$ with the decreases its rate from one sixth to one seventh in the training sample. The ${ }^{8} \mathrm{He} /{ }^{9} \mathrm{Li}$ background discrimination decreases from $51.8 \%$ to $39.9 \%$ with the decrease of its rate from one sixth to one seventh in the training sample. As a result, the most neutrino events and uncorrelated background events in the signal region can be identified with BNN, and the part events each of the fast neutron and ${ }^{8} \mathrm{He} /{ }^{9} \mathrm{Li}$ backgrounds in the signal region can be identified with BNN. The different signal 
to noise ratios in signal region are obtained with BNNs trained by the training samples consisting of neutrino events and background events at different rates in the reactor neutrino experiments. In a word, the signal to noise ratio in signal region can be enhanced with BNN in the reactor neutrino experiments.

\section{Acknowledgements}

This work is supported by the National Natural Science Foundation of China (NSFC) under the contract No. 10605014.

\section{References}

[1] R. M. Neal, Bayesian Learning of Neural Networks. New York: SpringerVerlag, 1996

[2] R. Beale and T. Jackson, Neural Computing: An Introduction, New York: Adam Hilger, 1991

[3] Y. Xu, J. Hou and K. E. Zhu, Chinese Physics C (HEP\&NP), 32(3), 201-204 (2008)

[4] Y. Xu, W. W. Xu, Y. X. Meng, and W. Xu, Nuclear Instruments and Methods in Physics Rearch A592, 451-455 (2008), arXiv: 0712.4042

[5] P. C. Bhat and H. B. Prosper Beyesian Neural Networks. In: L. Lyons and M. K. Unel ed. Proceedings of Statistical Problems in Particle Physics, Astrophysics and Cosmology, Oxford, UK 12-15, September 2005. London: Imperial college Press. 2006. 151-154

[6] S. Duane, A. D. Kennedy, B. J. Pendleton and D. Roweth, Physics Letters, B195, 216-222 (1987)

[7] M. Creutz and A. Gocksch, Physical Review Letters, 1989 63, 9-12

[8] P. B. Mackenzie, Physics Letters, 1989 B226, 369-371

[9] Daya Bay Collaboration, Daya Bay Proposal: A Precision Measurement of the Neutrino Mixing Angle $\theta_{13}$ Using Reactor Antineutrino At Daya Bay, arXiv: hep-ex/0701029

[10] F. Ardellier et al., Double Chooz: A Search for the Neutrino Mixing Angle $\theta_{13}$, arXiv: hep-ex/0606025

[11] Geant4 Reference Manual, vers. 9.0 (2007)

[12] The CHOOZ Experiment Proposal (1993), available at the WWW site http://duphy4.physics.drexel.edu/chooz_pub/

[13] Y. X. Sun, J. Cao, and K. J. Luk, et al., HEP \& NP, 29(6), 543-548 (2005)

[14] M. Apollonio et al., European Physical Journal C27, 331 (2003) 
Tab. 1: The different identification efficiencies are obtained with the BNNs trained by the different training samples, which consist of the neutrino and three backgrounds at different rates. The term after \pm is the statistical error of the identification efficiencies. The 3000 events each of the uncorrelated background, fast neutron and ${ }^{8} \mathrm{He} /{ }^{9} \mathrm{Li}$ are regarded as the test sample.

\begin{tabular}{|c|c|c|c|c|c|}
\hline neutrino(events) & 36000 & 30000 & 24000 & 24000 & 36000 \\
uncorrelated background(events) & 8000 & 8000 & 8000 & 4000 & 4000 \\
fast neutron(events) & 8000 & 8000 & 8000 & 8000 & 8000 \\
${ }^{8} \mathrm{He} /{ }^{9} \mathrm{Li}($ events) & 8000 & 8000 & 8000 & 8000 & 8000 \\
\hline neutrino eff.(\%) & $89.5 \pm 0.56$ & $86.7 \pm 0.62$ & $82.6 \pm 0.69$ & $85.0 \pm 0.65$ & $91.2 \pm 0.52$ \\
uncorrelated background eff.(\%) & $81.8 \pm 0.70$ & $84.6 \pm 0.66$ & $88.2 \pm 0.59$ & $82.6 \pm 0.69$ & $73.6 \pm 0.80$ \\
fast neutrons eff.(\%) & $39.2 \pm 0.89$ & $42.4 \pm 0.90$ & $48.5 \pm 0.91$ & $45.7 \pm 0.91$ & $37.6 \pm 0.88$ \\
${ }^{8} \mathrm{He} /{ }^{9} \mathrm{Li}$ eff.(\%) & $40.8 \pm 0.90$ & $44.8 \pm 0.91$ & $51.8 \pm 0.91$ & $49.9 \pm 0.91$ & $39.9 \pm 0.89$ \\
\hline
\end{tabular}




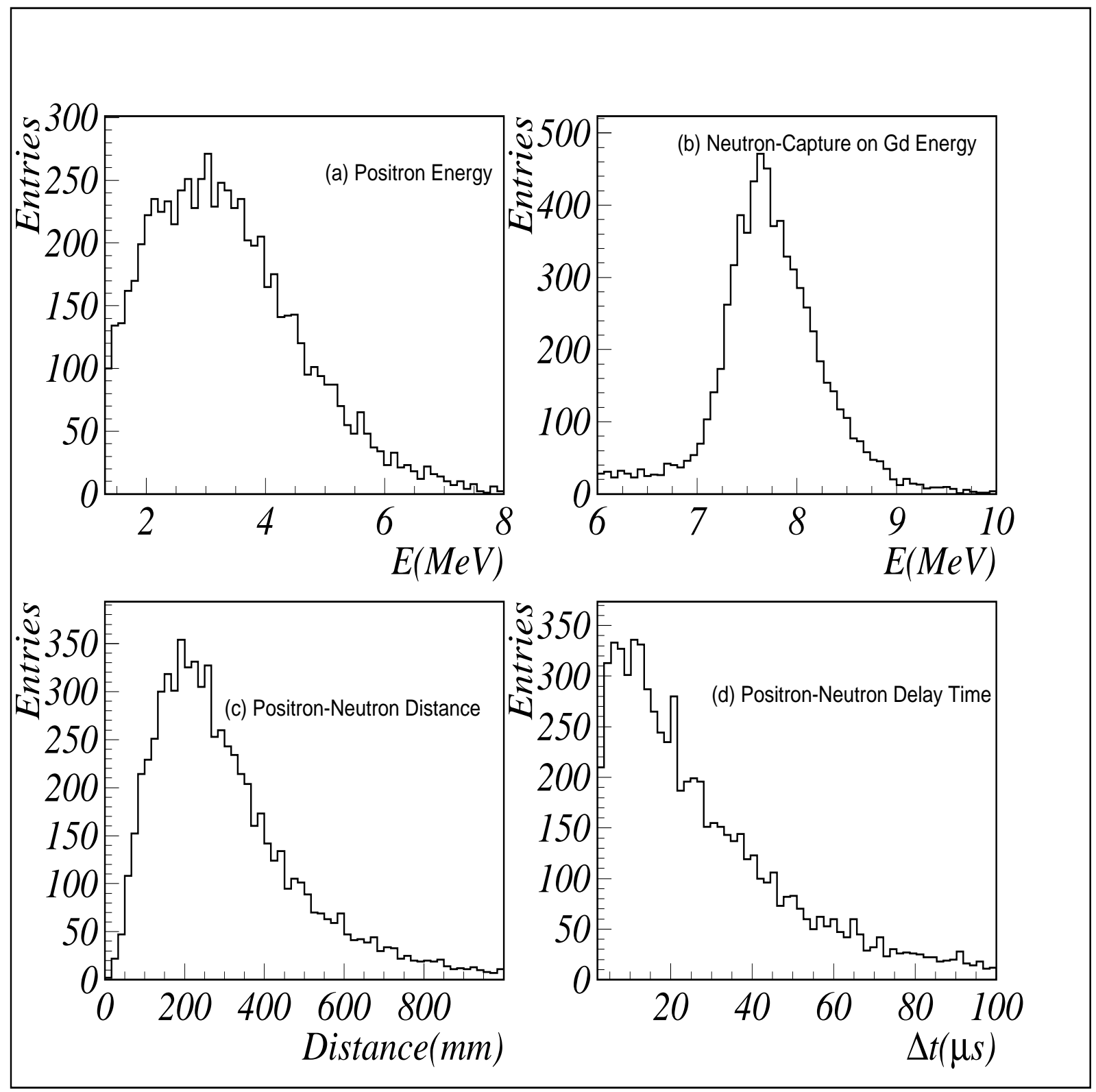

Fig. 1: The neutrino events for the Monte-Carlo simulation of the toy detector are uniformly generated throughout Gd-LS region. (a) is the distribution of the positron energy; (b) is the distribution of the energy of the neutron captured by $\mathrm{Gd}$; (c) is the distribution of the distance between the positron and neutron positions; (d) is the distribution of the delay time of the neutron signal. 